Cahiers $d u$ MONDE RUSSE

\section{Cahiers du monde russe}

Russie - Empire russe - Union soviétique et États indépendants

$50 / 4 \mid 2009$

Varia

\title{
Simo Mikkonen, Music and Power in the Soviet 1930s
}

Igor Contreras Zubillaga

\section{OpenEdition \\ Journals}

Édition électronique

URL : https://journals.openedition.org/monderusse/7170

DOI : 10.4000/monderusse. 7170

ISSN : $1777-5388$

\section{Éditeur}

Éditions de l'EHESS

\section{Édition imprimée}

Date de publication : 15 décembre 2009

Pagination : 814-817

ISBN : 978-2-7132-2261-0

ISSN : $1252-6576$

Référence électronique

Igor Contreras Zubillaga, "Simo Mikkonen, Music and Power in the Soviet 1930s », Cahiers du monde russe [En ligne], 50/4 | 2009, mis en ligne le 12 janvier 2011, consulté le 03 septembre 2022. URL : http://journals.openedition.org/monderusse/7170; DOI : https://doi.org/10.4000/monderusse.7170

Ce document a été généré automatiquement le 3 septembre 2022

Tous droits réservés 


\title{
Simo Mikkonen, Music and Power in the Soviet 1930s
}

\author{
Igor Contreras Zubillaga
}

\section{RÉFÉRENCE}

Simo MIKKONEN, Music and Power in the Soviet 1930s. A History of Composers'

Bureaucracy. Lewiston, NY : Edwin Mellen Press, 432 p.

1 L'étude de la vie musicale de l'ancienne URSS s'est considérablement renouvelée ces dernières années, notamment après l'ouverture des archives de l'État en 1991. La production musicale sous la dictature stalinienne a longtemps été considérée uniquement sous l'angle idéologique, comme un outil de propagande parmi d'autres. Les premiers travaux sur le sujet ont surtout montré comment le régime avait mis au pas le domaine artistique, détruisant de facto l'indépendance des artistes au profit d'une mise sous tutelle de la création. Face au pouvoir, l'artiste semblait devenir un sujet fonctionnel, figure malléable que l'on pouvait instrumentaliser à son gré ${ }^{\text {. Des recherches }}$ plus récentes ont quelque peu nuancé ce constat, montrant entre autres les dispositifs complexes qui régulaient la création artistique ${ }^{2}$. L'ouvrage de Simo Mikkonen s'inscrit dans cette tentative de reconsidération du fait culturel sous le régime soviétique. S'appuyant sur des sources d'archives, Mikkonen reconstitue le contexte administratif dans lequel évoluaient les compositeurs et les musicologues des années 1930. Il enquête sur leurs liens avec les acteurs du monde musical et les instances intermédiaires et supérieures du parti pour essayer d'élucider le degré d'autonomie et de dépendance des organisations chargées des affaires musicales et leur implication dans la formulation et la constitution du paradigme de la musique soviétique.

2 Les années 1930 sont sans doute une période particulièrement complexe dans l'histoire culturelle de l'URSS. En effet, elles se caractérisent à la fois par un interventionnisme politique dans la sphère artistique et par un essor créatif spectaculaire. Le 23 avril 1932, l'État dissout toutes les organisations artistiques existantes et les remplace par l'Union 
des compositeurs, organisation tristement connue pour la campagne menée en 1948 contre des compositeurs comme Šostakovič, Prokof'ev, Hačaturjan, Šebalin, Mjaskovskij et Popov, accusés de formalisme. Pourtant, cette décennie est aussi celle d'un développement extraordinaire du domaine musical, tant professionnel qu'amateur, avec de nouvelles créations et la consécration de compositeurs et interprètes qui remporteront les premiers prix des compétitions internationales les plus prestigieuses. Ce prétendu paradoxe entre l'institutionnalisation du contrôle idéologique et l'éclosion créative est l'un des points que tente d'élucider Mikkonen. Pour l'auteur, même si la résolution du 23 avril 1932 facilite la mainmise étatique sur les arts par le biais d'organismes récemment créés, les modalités d'application de ce contrôle n'ont pas été les mêmes dans toutes les disciplines. Mikkonen soutient que la décision de dissoudre toutes les organisations artistiques visait en priorité le domaine de la littérature. Les choses se seraient donc passées différemment dans le monde musical; au début des années 1930, le contrôle de la création musicale ne constituait pas une priorité pour le parti. Ainsi, à ses débuts, l'Union des compositeurs soviétiques n'était pas une institution centralisée, son programme ne faisait pas l'objet d'un contrôle strict des hautes instances politiques. En conséquence, contrairement à son pendant chez les écrivains, l'Union des compositeurs manquait d'organes nationaux, et ne fonctionnait qu'à travers des branches locales faiblement politisées. Mikkonen signale qu'en septembre 1933, la branche de Moscou - la plus importante avec celle de Leningrad - comptait 150 membres (105 compositeurs et 45 musicologues), dont seulement 10 étaient membres du parti ; même si, dans les premières années, ces derniers occupaient les postes à responsabilité, ils n'ont jamais réussi à véritablement imposer leur programme politique au sein de l'organisation. Pendant les années 1930, compositeurs et musicologues jouissaient d'une marge de manœuvre qui n'avait pas d'équivalent dans les autres domaines artistiques. Cette singularité était principalement la conséquence du mécanisme de prise de décision propre à l'Union de compositeurs. Ainsi, du fait d'une très faible ingérence du parti et des autres instances étatiques, l'Union a pu contrôler et distribuer ses ressources financières et surtout développer une politique qui visait à l'amélioration de la situation économique de ses membres, à travers la gestion des droits d'auteur et par le biais d'un système de bourses et de privilèges, qui incluait la mise à disposition d'appartements et de maisons de vacances. Le résultat est, et ce point constitue sans doute l'une des thèses les plus fortes du livre de Mikkonen, que l'Union des compositeurs, loin de se transformer en un instrument du parti, permit à ses membres de favoriser leurs propres intérêts professionnels et de se consacrer à temps plein à leur art.

3 L'Union des compositeurs n'était pourtant pas totalement imperméable à la réalité politique de l'époque. Mikkonen rappelle que les compositeurs et les musicologues de cette organisation prenaient une part très active dans les diverses tentatives de définir la nature de la culture soviétique, présentant volontiers l'opéra, le ballet, et les concerts de musique classique comme des genres officiels. Ainsi, la revue Sovetskaja Muzyka, contrôlée par l'Union, publie des reportages sur des nomades d'Asie centrale, la Sibérie ou encore le milieu rural, où l'on souligne le désir d'écouter des opéras nationalistes et d'assister à des concerts de musique proprement soviétique. Pourtant, l'auteur soutient que ces demandes étaient minoritaires dans les couches populaires, plus intéressées par les variétés, le jazz ou la musique de films - genre pour lequel le parti avait la plus haute estime ; et que ces revendications provenaient des compositeurs membres de différentes 
branches de l'Union, intéressés par la promotion de ces genres musicaux érudits, plutôt que de la population.

4 Au milieu des années 1930, la sphère musicale subit cependant une restructuration importante, avec la création du Comité des affaires artistiques. Fonctionnant comme une sorte de ministère, ce comité assure le contrôle de la musique, du cinéma, du théâtre et des arts plastiques, devenant ainsi l'organisme administratif culturel le plus puissant de l'Union soviétique. De plus, entre 1936 et 1938, la société soviétique est marquée par les purges staliniennes qui se soldent par plus de deux millions de victimes (dont 725000 exécutions). Pourtant, contrairement à l'Union des écrivains, l'Union des compositeurs connaît un nombre d'arrestations très faible. Mikkonen affirme même que la musique " semble presque avoir été un havre miraculeux au milieu de la Terreur, tandis que la littérature soviétique subissait de lourdes pertes » (p. 293). L'auteur ne cherche toutefois pas à minimiser l'expérience traumatique de compositeurs comme Šostakovič dont l'opéra Lady Macbeth de Mcensk, après avoir été écouté par Stalin, fut durement critiqué dans la Pravda, donnant lieu à toute une série d'accusations, défenses, et contre-attaques rituelles qui finiront par ostraciser l'œuvre pendant de longues années. Mais Mikkonen ne veut pas généraliser le cas particulier de Šostakovič. Aussi, après avoir examiné la situation de l'ensemble des membres de l'Union des compositeurs, il montre que malgré les querelles internes au sein de l'institution, ceux-ci réussirent à user de leur influence dans un contexte menaçant, protégeant même les anciens membres d'organisations prolétariennes qui se trouvaient au cœur des attaques de 1937. Ainsi, l'Union des compositeurs serait sortie de l'épisode des purges encore plus forte et indépendante, dirigée par des compositeurs plutôt que par des bureaucrates, et devenant après 1939 limite chronologique de l'enquête - une organisation de niveau national.

5 L'étude approfondie et détaillée de Simo Mikkonen réussit le pari de remettre en question le monolithisme et l'idée de «totalitarisme» musical avancée dans certains travaux antérieurs, permettant ainsi de mieux comprendre le contexte administratif de la création musicale soviétique des années 1930 . Toutefois, son ouvrage reste muet quant aux œuvres produites par cette bureaucratie, même s'il mentionne rapidement des créations qui avaient bénéficié d'aides octroyées par l'Union, comme la Douzième Symphonie de Mjaskovskij, la Suite Lenin de Šebalin, la Quatrième Symphonie (Poème pour les soldats du Komsomol) de Knipper, ou la Première Symphonie (antifasciste) de Mokrusov. S'intéresser davantage à ce genre de corpus, aussi bien d'un point de vue esthétique que politique, aurait sans doute enrichi considérablement l'étude de la bureaucratie soviétique des années 1930, en apportant de nouveaux éléments à la question de la participation des compositeurs dans la culture soviétique, et en intégrant notamment les gestes compositionnels dans la description du positionnement des créateurs par rapport au pouvoir. Cette perspective heuristique pourrait être intéressante à exploiter dans de futures recherches et permettrait sans doute de mieux appréhender une période historique aussi riche, complexe et controversée. 


\section{NOTES}

1. Voir par exemple le très célèbre ouvrage de Boris Schwarz, Music and Musical Life in Soviet Russia, 1917-1981, Bloomington, IN : Indiana University Press, 1983.

2. Voir notamment: Neil Edmunds, The Soviet Proletarian Music Movement, Berne-New YorkOxford: Peter Lang, 2000 ; Neil Edmunds, éd., Soviet Music and Society under Lenin and Stalin: The Baton and Sickle, Londres-New York: RoutledgeCurzon, 2004; Amy Nelson, Music for the Revolution: Musicians and Power in Early Soviet Russia, University Park: Pennsylvania State University Press, 2004; Kiril Tomoff, Creative Union. The Professional Organization of Soviet Composers, 1939-1953, Ithaca-Londres: Cornell University Press, 2006 ; Marina Frolova-Walker, Russian Music and Nationalism from Glinka to Stalin, New Haven-Londres : Yale University Press, 2007. 